\title{
Schülerinnen im Gymnasium: ein interkantonaler Vergleich der ungleichen Beteiligung von Mädchen in der Schweiz im 20. Jahrhundert
}

\author{
Thomas Ruoss und Lars Heinzer, Universität Zürich
}

\begin{abstract}
Dieser Artikel stellt die Entwicklung der Mädchenbeteiligung in der gymnasialen Sekundarstufe II in der Schweiz während des 20. Jahrhunderts dar. Dazu kann er auf eine umfangreiche Grundlage statistischer Langzeitdaten zurückgreifen. In einem ersten Schritt werden die Unterschiede zwischen den Kantonen dargestellt und drei Phasen der Beteiligung von Mädchen im Gymnasium hergeleitet. In einem zweiten Schritt werden die festgestellten Unterschiede mit Blick auf die strukturelle Differenzierung der Gymnasien untersucht und die Bedeutung institutioneller Rahmenbedingungen für Geschlechterdifferenzen in der Schule diskutiert.
\end{abstract}

\section{Einleitung}

Die gymnasiale Sekundarstufe II ist in den letzten Jahren erneut zu einem bildungspolitischen Konfliktfeld avanciert. Übertritte und kantonal unterschiedliche Eintrittsbedingungen, Drop-outs, sowie Fragen der «Studierfähigkeit» werden in einer breiten Öffentlichkeit diskutiert (Eberle, 2018). Wenig überraschend standen im letzten Bildungsbericht Schweiz (SKBF, 2018, S. 140f.) die Maturitätsquoten im Zentrum der Analysen zum Gymnasium. Neben Fragen zu den erheblichen interkantonalen Unterschieden und den schicht- und herkunftsspezifischen Faktoren für die Gestaltung individueller Bildungsverläufe, wurde als weiterer Einflussfaktor für die Wahrnehmung von Bildungschancen auch die Bedeutung von Geschlecht thematisiert. «Frauen am Gymnasium» werden im Bildungsbericht nicht nur aus der Perspektive von Chancengerechtigkeit betrachtet, sondern als historisch mitentscheidender Faktor für das Wachstum der Maturitätsquoten seit der Bildungsexpansion verstanden. Gesamtschweizerisch erwarben erstmals im Jahr 1993 mehr Frauen eine Maturität und bis im Jahr 2016 stieg die gymnasiale Maturitätsquote der Frauen auf 23.7\%, während diejenige der Männer bei $15.7 \%$ lag (ebd.) ${ }^{1}$. In sämtlichen Kantonen ist die Zahl der Frauen in den Gymnasien inzwischen höher als diejenige der Männer. Gleichzeitig zeigen sich nach wie vor grosse kantonale Unterschiede sowohl bei der Maturitätsquote als auch bei den Geschlechterverhältnissen. Der Bildungsbericht hält dazu fest, dass die Frage "welche Faktoren diese Unterschiede zwischen den Kantonen bestimmen, [...] nie genauer untersucht worden [sei]» (ebd., S. 141).

Dieser Befund lässt aufhorchen und er trifft sich mit der Feststellung, dass die Forschung sich zwar mit unterschiedlichen Aspekten der Mädchenbildung in der Schweiz befasst hat, eine kantonal vergleichende, und über einen längeren Zeitraum angelegte und auf breiter Datenbasis gestützte Analyse der gymnasialen Mädchen-beteiligung bislang noch nicht vorgelegt wurde (Zangger \& Becker, 2016). In der internationalen Forschung wird ebenfalls vermehrt ein historischer Blick auf unterschiedliche Entwicklungen der Mädchenbeteiligung in der Sekundarstufe II eingefordert (Albisetti, 2010, S. 7). Damit sollen die historisch gewachsenen institutionellen und kulturellen Rahmenbedingungen von Schule und Unterricht stärker berücksichtigt (Giudici \& Manz, 2018) und damit ein Beitrag geleistet werden zur Frage, wie sich Geschlechterdifferenzen und Bildungschancen durch diese Rahmenbedingungen beeinflussen lassen. Allerdings liegen, mit Blick auf die internationale Forschung von einigen, teilweise älteren Arbeiten abgesehen (Mayeur, 1977; Lelièvre \& Lelièvre, 1991; Zymek et al., 2005; Rogers, 2010), kaum historisierende, quantifizierende Forschungsergebnisse zum Geschlechterverhältnis in den Gymnasien vor (Martin \& Goodman, 2011). Das Erkenntnispotenzial sozialhistorischer Methoden und die Nutzung der deskriptiven historischen Statistik lagen lange

1 Bei der 1994 eingeführten Berufsmaturität glich sich ein zunächst deutlich höherer Anteil von Männern allmählich aus. Der Frauenanteil an Berufsmaturitäten stabilisierte sich seit 2010 auf zwischen 46\% und 48\%. Die Berufs- und Fachmaturitäten, die erst im letzten Zeitabschnitt dieser Analyse entstanden sind, werden nachfolgend nicht berücksichtigt. 
brach. Mit Blick auf die über das gesamte 20. Jahrhundert stark steigende Mädchenbeteiligung stellt sich uns daher zunächst die Frage: Wie veränderte sich die Mädchenbeteiligung in der gymnasialen Sekundarstufe II im interkantonalen Vergleich in der Schweiz im Verlauf des 20. Jahrhunderts? Inwiefern gingen strukturelle Differenzierungen innerhalb der gymnasialen Sekundarstufe II mit einer veränderten Mädchenbeteiligung einher?

Mittels einer vergleichenden Langzeitanalyse der gymnasialen Mädchenbeteiligung in verschiedenen Kantonen der Schweiz wollen wir grundlegendes Wissen zur Entwicklung des Bildungssystems erarbeiten und darüber hinaus die Rolle der Systemdifferenzierung für die Mädchenbeteiligung diskutieren.

Der Artikel ist folgendermassen aufgebaut: Nach dieser Einleitung werden in Kapitel 2 sowohl der Forschungsstand aufgearbeitet und die damit verbundenen theoretischen Annahmen für die zwischen den Kantonen divergierenden Entwicklungen hergeleitet. In Kapitel 3 wird das methodische Vorgehen der empirischen Analyse expliziert sowie die Fallauswahl und die untersuchten Quellen besprochen. In Kapitel 4 werden die Daten zur kantonalen Mädchenbeteiligung auf ihre allgemeine Entwicklung hin untersucht. Auf dieser Ebene allgemeiner Entwicklungen können wir sowohl die gegenwärtigen Differenzen in ein historisches Verhältnis setzen als auch die Bedeutung eines spezifischen Blickes für die kantonalen Differenzen deutlich machen. In Kapitel 5 werden die allgemeinen und langfristigen historischen Entwicklungen auf ihre Spezifika befragt und die Frage nach der strukturellen Differenzierung des Gymnasiums im Verlaufe der Zeit untersucht. Schliesslich wird in Kapital 6 die Frage der Finanzierung der Mittelschulen als Voraussetzung der Bildungsexpansion in die Analyse miteinbezogen. Die Ergebnisse werden in einem Schlusskapitel diskutiert und auf mögliche Anschlussfragen hingewiesen.

\section{Die Entwicklung des Gymnasiums und der Blick auf Geschlechterdifferenzen: zum Forschungsstand}

Die Geschichte des Gymnasiums in der Schweiz kann entlang dreier Entwicklungen beschrieben werden: 1) der schrittweisen Hinwendung zu «Realien» und damit der Differenzierung des Fächer- und Typenkanons seit dem letzten Drittel des 19. Jahrhunderts, 2) der «Bildungsexpansion» der 1960er-Jahre und damit der Vervielfachung und Dezentralisierung des Angebots an gymnasialem Unterricht mit dem zweifachen Ziel der Förderung der Chancengerechtigkeit sowie dem Entgegenwirken des sog. Nachwuchsmangels in höher qualifizierten Berufen, und 3) der Neuordnungen der Sekundarstufe II in den 1990er-Jahren mit der Einführung von Berufs- und Fachmaturität sowie der Auflösung der Maturitätstypen (Criblez, 2001; 2002; Vonlanthen et al., 1978). Einerseits wurde die Geschichte des Gymnasiums in der Schweiz bislang noch kaum als eine Geschichte der Mädchenbildung verstanden. Andererseits lässt sich die historische Forschung zur Mädchenbildung in der Schweiz gliedern in Biographien und Personengeschichten (z.B. Joris, 2011), in Institutionengeschichten (z.B. Hodler, 2014; Crotti, 2005) sowie Studien zur Konstruktion von Weiblichkeit in und durch die Schule via Lehrmittel und Lehrpläne (z.B. Giudici \& Manz, 2018; Kellerhals, 2010; Mantovani Vögeli, 1994). Erklärungsangebote zum Zusammenhang der Entwicklung der Sekundarstufe II und des Geschlechterverhältnisses sind folglich rar.

Die zentrale Voraussetzung für die Beteiligung von Mädchen im Gymnasium ist deren Zulassung als Schülerinnen dieser Bildungsstufe, die in vielen Kantonen schrittweise um 1900 ermöglicht wurde (EKF, 2001; Ullmann, 2019). Darüber hinaus wird (international) die strukturelle Differenzierung innerhalb von Schulsystemen als eine Folge des politischen Kampfes um schulische, gesellschaftliche und berufliche Alternativen für Mädchen und Frauen beschrieben (Zymek et al., 2005; Jacobi, 2013). Auch für die Schweiz wird der Rolle der geschlechtsspezifischen gesellschaftlichen Rollen- und Berufsperspektiven für die Begründung zur Absolvierung einer Ausbildung auf der Sekundarstufe II eine zentrale Bedeutung zugemessen (Renold, 1998). So wurde den Frauen die Chance für wirtschaftliche Karrieren durch höhere Bildung lange gezielt strukturell verunmöglicht. Noch 1934 verweigerte etwa die Stadt Zürich der Höheren Töchterschule die Einführung einer Handelsmaturität mit dem Verweis auf die zunehmende Konkurrenz auf dem Arbeitsmarkt (EKF, 2001, S. 8). Von diesem Beispiel ausgehend, gilt es für den kantonalen Vergleich, die in der politikwissenschaftlichen Literatur als Cleavages bezeichneten Strukturmerkmale (Lipset, 1967), insbesondere die Bevölkerungs- und die Wirtschaftsstruktur, zu berücksichtigen.

Die Frage der Zulassung geht daher eng einher mit derjenigen der strukturellen Differenzierung innerhalb des Schulsystems. Als Nukleus für die erhöhte Mädchenbeteiligung wird folglich gerne die Bildungsexpansion der 1960/70er-Jahre verstanden. Der starke Mittelschulausbau dieser Zeit sei zwar programmatisch «geschlechtsblind» erfolgt und schöpfte dennoch von den «Mädchen als Hauptreservoir, [...] mit dem niemand gerechnet hatte» (Rieger, 2001, S. 55). Mit der Bildungsexpansion hat sich auch die Konkurrenz zwischen 
unterschiedlichen Bildungsangeboten im nachobligatorischen Bereich - insbesondere zwischen dem allgemeinbildenden und dem berufsbildenden Bereich - zugespitzt. Während wir gegenwärtig von beträchtlichen kantonalen Unterschieden im Geschlechterverhältnis zwischen allgemein- und berufsbildenden Bildungsverläufen ausgehen können (Imdorf et al., 2014), zeigt sich gesamtschweizerisch eine relative hohe historische Konstanz des Geschlechterverhältnisses innerhalb der berufsbildenden Sekundarstufe II seit der Bildungsexpansion (Borkowsky, 2000). Allerdings ist der Forschungsstand zur sich wandelnden Bedeutung des Geschlechts im Kontext der beruflichen Bildung für die Schweiz sehr lückenhaft. Dies ist umso erstaunlicher, da Geschlecht als der stärkste personale Prädiktor für (ausbleibende) ökonomische Aufwärtsmobilität in der Schweiz angesehen werden kann (Chuard \& Grassi, 2020). Eine Differenzierung innerhalb der gymnasialen Bildungsgänge während der Bildungsexpansion, so die daraus hergeleitete These, sollte zu einer höheren, respektive äquivalenteren Mädchenbeteiligung führen.

Mittelschulausbau und Systemdifferenzierung sind nur mit dem politischen Willen zur Finanzierung dieser Entwicklungen möglich. Höhere Investitionen in Bildung lassen auf ein gut finanziertes und damit weniger ausschliessendes Bildungssystem schliessen (Becker \& Lauterbach, 2016 ; Ragni, 2014). Bereits in der Phase der formalen Zulassung von Mädchen zu den Gymnasien im frühen 20. Jahrhundert war das Aufbringen der zusätzlichen Finanzen eines der zentralen Argumente gegen deren Öffnung (Ullmann, 2019). Entsprechend kann ein Vergleich der kantonalen Bildungsausgaben für die Mittelschulen einen zusätzlichen Hinweis bieten für die politische Bereitschaft, die Expansion - und damit die Mädchenbeteiligung - voranzutreiben.

Das schulische Angebot ermöglicht und limitiert zugleich individuelle Laufbahnentscheide, nimmt diese hingegen nicht vorweg. Vielmehr sind Laufbahnentscheide innerhalb eines differenzierten Schulsystems abhängig von den individuellen Erwartungen der Schülerinnen in ihren schulischen und beruflichen Erfolg (McDaniel, 2010) sowie den Kosten-, Nutzen- und Erfolgserwartungen an höhere Bildung der Eltern für die Laufbahnentscheidungen ihrer Kinder (Breen et al., 2000; Helbig, 2013). Diese Erwartungen gründen wiederum wesentlich auf den antizipierten gesellschaftlichen Rollen der Geschlechter (Jacobi, 2013). Da sich die individuell antizipierten Rollenmuster im Laufe der persönlichen Entwicklung verändern, wird auch dem Selektionszeitpunkt für eine allgemein- oder berufsbildende Laufbahn ein Einfluss auf die Geschlechterdifferenz zugeschrieben (Imdorf et al., 2014).

Mit Blick auf die historische Entwicklung ist der Wandel der kulturellen und gesellschaftlichen Geschlechterund Rollenbilder von Bedeutung - so war beispielsweise die Legitimation höherer Bildung für Mädchen innerhalb des Bürgertums primär als Statussymbol zu verstehen (Albisetti, 2010, S. 2). Die preussischen Schulreformen von 1880-1920, denen eine Vorbildfunktion für ganz Kontinentaleuropa zugesprochen wird, verbanden die höhere Bildung für Mädchen nicht zwingend mit der Zulassung an weiterführende Schulen, sondern boten einen spezifisch weiblichen Lehrplan, der die Mädchen auf ihre zukünftige Rolle als Ehefrauen und Mütter von studierten bürgerlichen Männern vorbereiten sollte (Jacobi, 2013, S. 346).

Zusammengefasst führt uns die Herleitung zu einem beträchtlichen Forschungsdesiderat betreffend dem Geschlechterverhältnis in der Sekundarstufe II. Dieser Artikel erhebt nicht den Anspruch, alle daraus entstehenden Hypothesen zu prüfen und die Forschungslücken zu schliessen. Vielmehr will er, mittels eines auf einer langen Zeitachse angelegten deskriptiv-explorativen Zugangs, einige zentralen Grundlagen erarbeiten, um die relevanten Thesen für die Schweiz zu konkretisieren. Darauf aufbauend steht die Frage im Zentrum, inwiefern eine Differenzierung innerhalb der gymnasialen Sekundarstufe II mit einer veränderten Mädchenbeteiligung einher geht. Prüfen wollen wir die These, dass im Rahmen des aktiven Mittelschulausbaus während der Bildungsexpansion die Mädchenbeteiligung flächendeckend gestiegen ist - und die damit verbundene Differenzierung innerhalb der gymnasialen Sekundarstufe II einen wesentlichen Beitrag dazu geleistet hat.

\section{Schülerinnen in Zahlen: Datengrundlage \& methodisches Vorgehen}

Unsere Analyse stützt sich empirisch auf deskriptive schulstatistische Daten zu den Gymnasien in der Schweiz. Diese Daten wurden im Rahmen eines Infrastrukturprojektes des Schweizerischen Nationalfonds (SNF 130398, 146239 \& 170407) inventarisiert, kommentiert und für die Analyse und Nachnutzung öffentlich zugänglich gemacht (www.bildungsgeschichte.ch). Die dahinterliegende Datengrundlage ist sehr breit, weshalb auf eine Verweisstruktur zu den Originalquellen verzichtet wird. In vielen Fällen wurden die Datensätze bis auf Ebene der einzelnen Schulen differenziert beigezogen. Dies war insofern notwendig, da historisch gesehen die meisten höheren Bildungsinstitutionen unterschiedliche Ausbildungsgänge angeboten haben (beispielsweise 
Lehrer*innenausbildung, Handelsdiplome, usw.) und nicht nur zur Erlangung eidgenössisch oder kantonal anerkannter Maturitätsdiplome geführt haben. ${ }^{2}$

Die Zäsur für unsere Analyse ist zunächst durch die formale Zulassung von Mädchen zum Gymnasium definiert, die in den ersten Kantonen um das Jahr 1900 de jure ermöglicht wurde (VD, BE, ZH, SO, SH, SG, AG; vgl. EKF, 2001) und in Folge kontroverser Diskussionen zu unterschiedlichen separativen oder ko-edukativen Modellen geführt hat (Ullmann, 2019). Für insgesamt 13 der 26 Kantone konnten für diesen Zeitraum valide und vergleichbare Daten zu den Lernenden der gymnasialen Mittelschulen differenziert nach Geschlecht rekonstruiert werden. Damit können keine individuellen Bildungslaufbahnen rekonstruiert, aber die Annahme einer Angleichung zwischen den geschlechtsspezifischen Unterschieden in den höheren Bildungsgängen (Zangger \& Becker, 2016) substantiell empirisch unterlegt werden. In sieben dieser Kantone waren darüber hinaus auch die Geschlechterverhältnisse nicht nur nach einzelnen Schulen, sondern bis auf Ebene der Bildungsgänge rekonstruierbar. Für die Frage nach dem Einfluss der Differenzierung des Schulsystems auf die Entwicklung der Mädchenbeteiligung sind diese Kantone besonders aufschlussreich. Für den Zeitraum nach 1980 - und damit seit der Nationalisierung der Schweizerischen Schulstatistik (Ruoss, 2013) - wurden Daten des Bundesamtes für Statistik beigezogen.

\section{Abbildung 1}

\section{Kantone mit geschlechterdifferenzierten Langzeitdaten der Gymnasien}

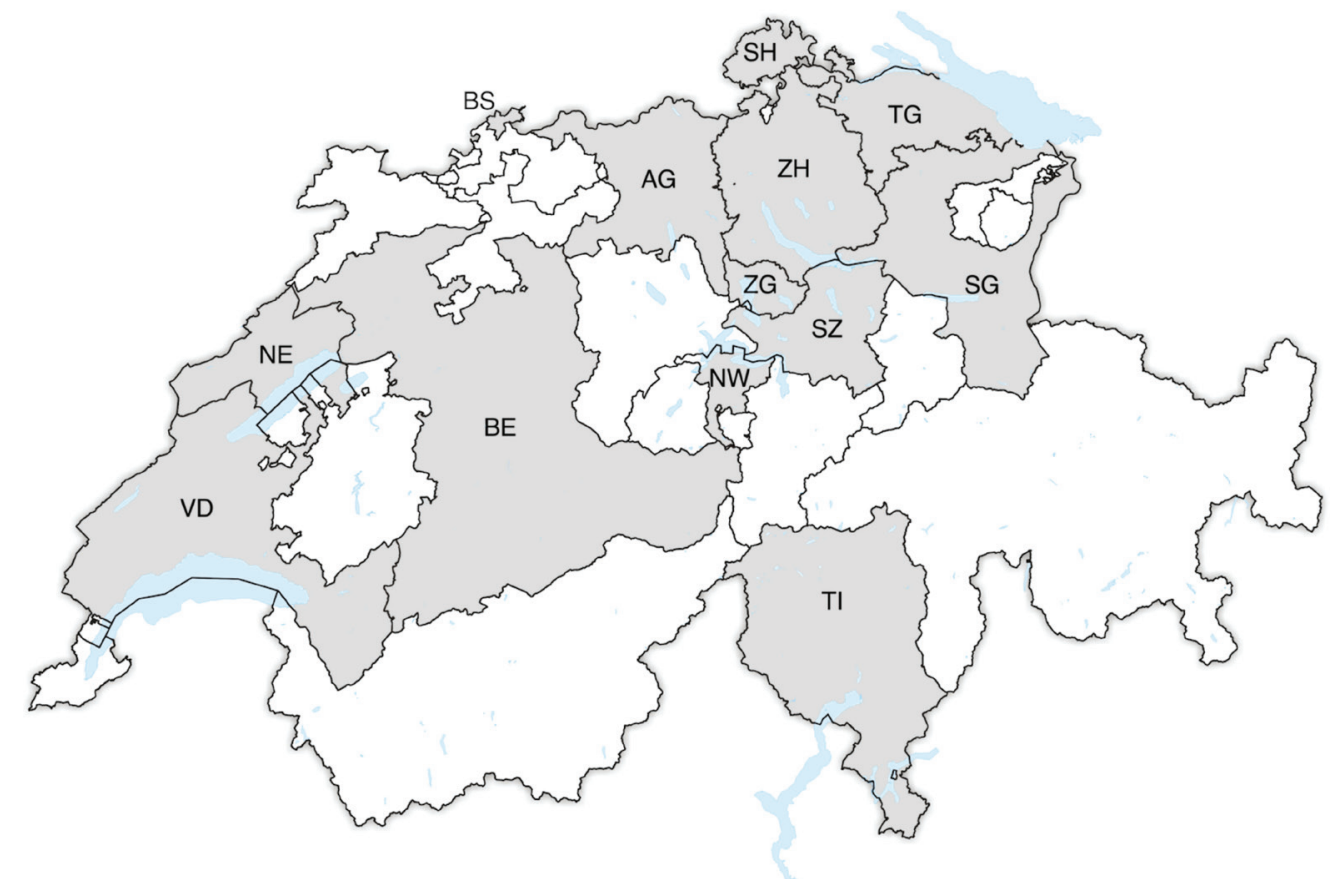

Bem. Mit Typendifferenzierung: AG, BE³, NE, TG, TI, ZG, ZH

Ohne Typendifferenzierung: BS, NW, SG, SH, SZ, VD

2 Die Datenreihen basieren auf jährlichen Vollerhebungen der Schüler*innen durch die Schulen selbst. In den Fällen, für die keine jährlichen Daten zugänglich waren oder sich einzelne Angaben als nicht valide herausgestellt haben (z.B. bei der Kopie von Daten aus dem vorangegangenen Schuljahr) wurden grafisch die bekannten Datenpunkte direkt miteinander verbunden. Die Kurven überbrücken fehlende Daten mit direkten Verbindungen von Datenpunkten. Ein Wert liegt bei 0, wenn nachweislich keine Mädchen eine Schule besucht haben.

Grosse Sprünge in den Datenreihen sind in den meisten Fällen erklärbar entweder mit Umstellungen der Zählpraktiken (z.B. sich verändernde Aggregatsgrade) oder mit institutionellen Neuordnungen (z.B. der Zulassung einer Handelsschule als Handelsmaturitätsschule).

3 Bis zum Schuljahr 1980/81 wurde der Kanton Jura als Teil des Kantons Bern gezählt. Die Zahl der Gymnasiast*innen ist in BE (für den gesamten Zeitraum nach 1980) um etwas 10x grösser als in JU. Das Geschlechterverhältnis fällt in JU im Jahr 1980/81 stärker zugunsten der Mädchen aus als in BE (44\% vs. 39\%). Im Schuljahr 2016/17 ist das Geschlechterverhältnis nahezu ausgeglichen. 


\section{Allgemeine Entwicklungen und die Bedeutung des Spezifischen}

Betrachten wir die Entwicklung der kantonalen Unterschiede bis anfangs des 20. Jahrhunderts im Rückblick, so lassen sich drei generelle Phasen ausmachen (Abb. 2): eine Phase der Disparität (bis 1980), eine Phase der Homogenisierung (zwischen 1980 bis 2000) und eine Phase der Konsolidierung (seit 2000).

1) Phase der Disparität: Die Dynamiken, die wir im Zeitraum vor 1980 feststellen, sind von einer langanhaltenden Disparität geprägt. Während dieser Phase zeichnen sich seinerseits drei unterschiedliche Typen ab: Kantone mit einer stabil hohen Mädchenbeteiligung (Typ «Stabilität»: VD, NE, BS), solche mit einem kontinuierlichen Wachstum (Typ «Wachstum»: AG, BE, SG, SH, TG, TI, ZG, ZH) und schliesslich zwei Kantone mit einer schnellen Öffnung in den 1970er-Jahren (Typ «späte Öffnung»: SZ, NW). Während eine grosse Gruppe an Kantonen mit unterschiedlichen strukturellen Ausprägungen dem mittleren Typus zuzuordnen ist, finden sich im ersten Typus protestantische Kantone mit starken wirtschaftlichen Zentren und im dritten Typus ländliche, katholische Gebirgskantone (vgl. Abb. 3). Allerdings lässt gerade die grosse und strukturell heterogene Gruppe des Typus kontinuierliches «Wachstum» darauf schliessen, dass hier wenig eindeutige Zusammenhänge zu erwarten sind.

Die Einsicht in diese, gerade im Vergleich zu den gegenwärtigen Unterschieden in der Mädchenbeteiligung, grossen Unterschiede zwischen den untersuchten Kantonen ermöglicht uns, unseren Blick für die gegenwärtige Situation in historische Relationen zu setzen. Insgesamt kann man gegenwärtig von einer historisch ausserordentlich homogenen Mädchenbeteiligung in den gymnasialen Mittelschulen in den (ganz unterschiedlichen) Kantonen sprechen.

2) Phase der Homogenisierung: Für den Zeitraum zwischen 1980 und 2000 stellen wir einerseits ein Wachstum der Mädchenbeteiligung in allen Kantonen fest, das sich aber seit 1980 auf deutlich unterschiedlichen Niveaus bewegt. Während VD und NE bereits früh mehr als 50\% Mädchenbeteiligung aufweisen, holen zunächst ZH und TI auf. Die Kantone SZ, ZG, SG, NW und TG entwickeln sich von einem tiefen Niveau her. Zwischen SZ (33\%) und NE (51\%) liegt im Jahr 1981 eine Differenz der Mädchenbeteiligung von 18\%. Im Jahr 1999/2000 findet sich die grösste Differenz zwischen ZG (45\%) und NE (58\%), alle anderen Kantone der Fallauswahl haben eine Mädchenbeteiligung von über 50\% erreicht.

3) Phase der Konsolidierung: Für das Schuljahr 2016/17 - wir erinnern uns, dass der Bildungsbericht hier die grossen Unterschiede zwischen den Kantonen betonte - zeigen sich die grössten Differenzen zwischen den Kantonen BS mit der tiefsten (54\%) sowie TG mit der höchsten (62\%) Mädchenbeteiligung. Im Zeitraum nach 2000 sind die entsprechenden kantonalen Unterschiede relativ stabil geblieben und bewegen sich in etwa innerhalb dieser 10\%-Differenz.

\section{Abbildung 2}

Gymnasiale Mädchenbeteiligung in den Kantonen

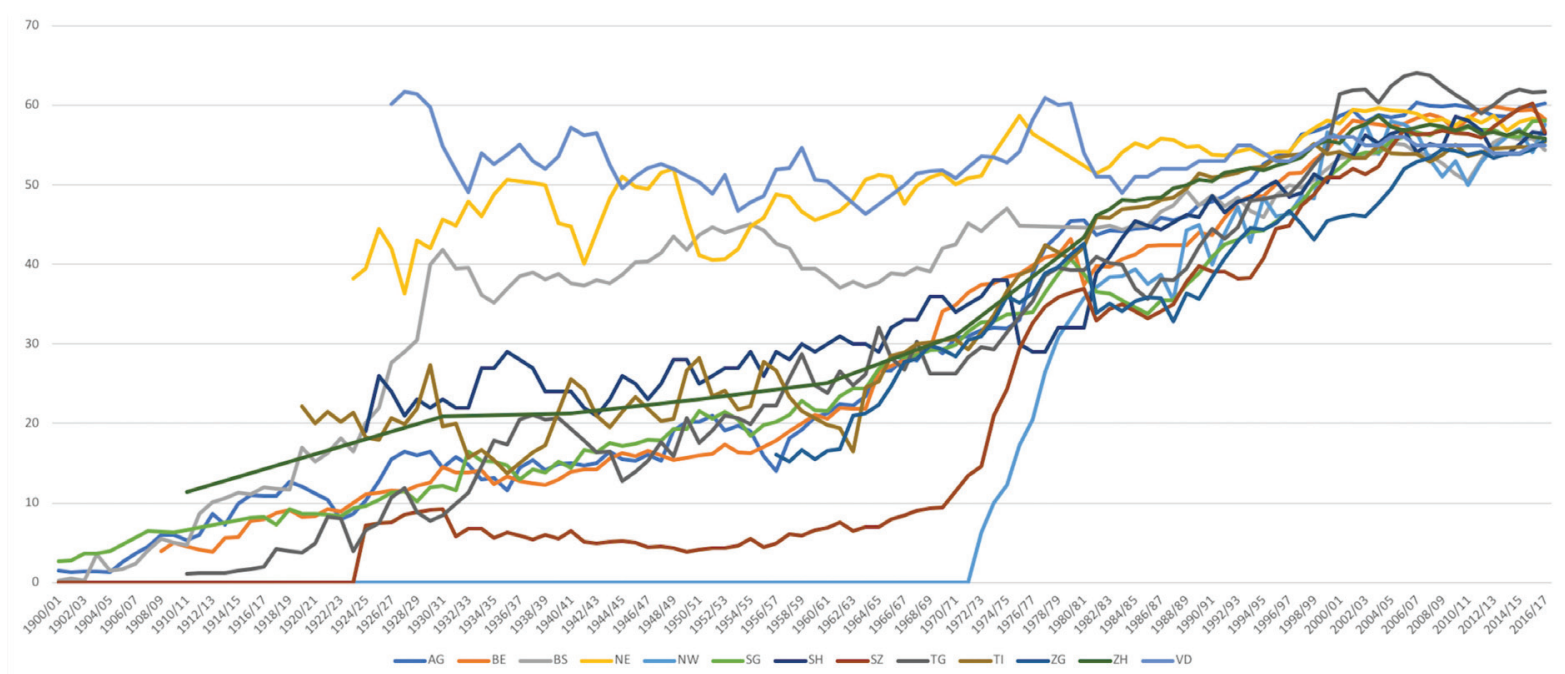

Bem. Daten für 13 Kantone in Prozent, 1900-2017. 
Bei diesem Vergleich bleibt festzuhalten, dass einige Kantone bis in die 1970er-Jahre eine Geschlechtersegregation der Ausbildungsgänge aufgewiesen haben. Dabei zeigt sich, dass ein traditionell ausgebautes aber geschlechter-segregiertes Modell in der Sekundarstufe II, wie es die Kantone BS, VS oder ZH kannten, sowohl die hohe Mädchenbeteiligung im Gymnasium, als auch die Persistenz der geschlechtersegregierten Strukturen beförderte. Dabei muss bedacht werden, dass in der bewussten Aufrechterhaltung dieser strukturellen Segregation auch potenziell diskriminierende Aspekte von Bedeutung waren (vgl. Ullmann, 2019). Das Ende der geschlechterspezifischen institutionellen Separation zwischen den maturitären Mittelschulen koinzidiert zeitlich jeweils mit einem Wachstum der Mädchenbeteiligung.

Wenn wir davon ausgehen, dass der Mittelschulausbau der 1960/70er-Jahre allgemein zu einem Schub der Mädchenbeteiligung geführt hat, lohnt es sich, die drei unterschiedlichen kantonalen Entwicklungstypen v.a. in der Phase der Disparität vergleichend zu betrachten. Die Bildungsexpansion - so lässt sich anhand dieses Vergleiches schliessen - hatte einen Einfluss auf die Mädchenbeteiligung in den Gymnasien. Dieser war besonders ausgeprägt in den vergleichsweise kleinen, ländlich geprägten Kantonen, die bis zu diesem Zeitraum gar kein (NW) oder nur ein kleines (SZ) Angebot an gymnasialen Mittelschulplätzen für Mädchen aufwiesen. Die Kantone des Typs kontinuierliches «Wachstum» erfuhren einen kleinen Schub in den Jahren 1975 - 1980. Für die Kantone mit einer stabil hohen Mädchenbeteiligung zeigte die Bildungsexpansion hingegen keinen wesentlichen Einfluss.

\section{Abbildung 3}

Gymnasiale Mädchenbeteiligung nach kantonalen Typen

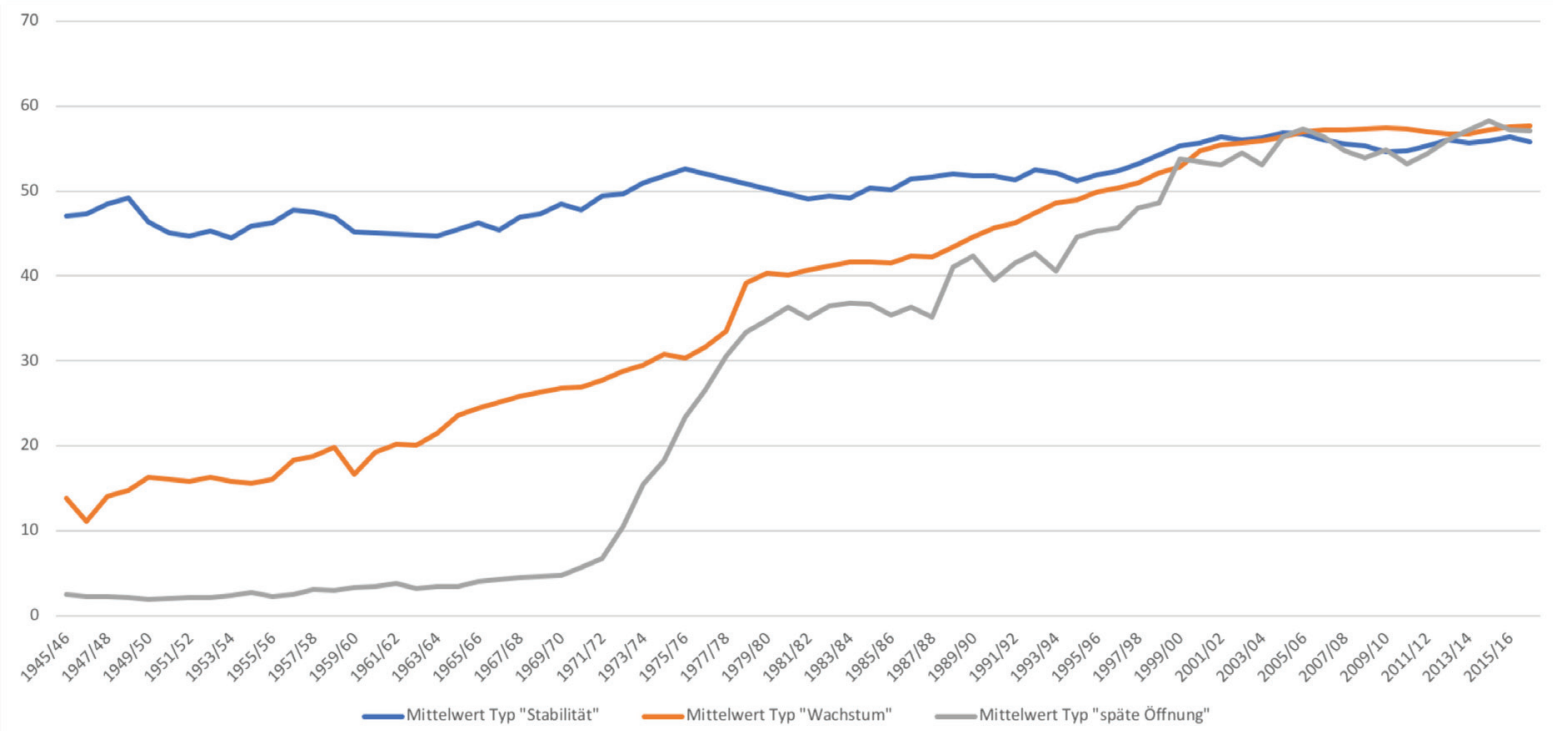

Bem. Daten als Mittelwerte in Prozent (Daten für $\mathrm{ZH}$ ab 1980).

\section{Strukturelle Differenzierungen des Gymnasiums}

\section{Typendifferenzierungen}

Die Bildungsexpansion zeichnete sich nicht einzig über einen Zuwachs an Gymnasien und Gymnasiast*innen aus, sondern auch über eine Differenzierung der gymnasialen Typen. Wir wollen daher auf die typendifferenzierten Langzeitdaten für sieben Kantone eingehen, für die entsprechende Daten vorliegen. Hieraus ergibt sich zunächst ein relativ klares Bild: Die Einführung neuer Maturitätstypen hängt nicht mit einer steigenden Mädchenbeteiligung zusammen. In allen untersuchten Kantonen bleibt die Mädchenbeteiligung im Typus $\mathrm{A} / \mathrm{B}^{4}$ für die Gesamtentwicklung prägend. Sie ist dabei sowohl vergleichbar zwischen den Kantonen als auch mit

4 Eidgenössisch anerkannte Gymnasialtypen: Typus A/B: altsprachlich-humanistisch; Typus C: mathematisch-naturwissenschaftlich; Seit 1972: Typus D: neusprachlich; Typus E: wirtschaftlichswissenschaftlich 
der durchschnittlichen Entwicklung der Mädchenbeteiligung im jeweiligen Kanton. Die Anerkennung neuer eidgenössischer maturitärer Typen $\mathrm{E}$ und $\mathrm{D}$ in den 1970er-Jahren führte tendenziell zu einer Umschichtung der Mädchenbeteiligung zwischen den Typen, und nicht zu einer generellen Erhöhung. Während die Mädchenbeteiligung in den Typen A, B und C weiterhin anstieg, konsolidierten die neuen Typen D (auf hohem) und E (auf unterdurchschnittlichem) Niveau. Das weitere Wachstum der Mädchenbeteiligung wurde seit Mitte der 1970er-Jahre nicht durch diese neuen Typen generiert. Die Entwicklung des Typus E zeichnete sich, für diejenigen Kantone, die entsprechende Daten aufweisen (AG, BE, TI, ZH), zunächst durch volatile Entwicklungen aus. Diese Schwankungen der Mädchenbeteiligung des Typus E innerhalb der Kantone waren über die Jahre gesehen beträchtlich, lagen aber nur punktuell über den kantonalen Mittelwerten. In der Einführung des Typus E kann daher kein Einfluss auf eine steigende Mädchenbeteiligung erkannt werden. Interessant ist hier das Verhältnis zwischen einzelnen Kantonen: Während im Vergleich zu anderen Typen in BE viele Mädchen den Typus E gewählt haben, finden wir beispielsweise in BS sowohl im Vergleich zu den anderen Kantonen und erst recht im Verhältnis zur Entwicklung innerhalb des Kantons BS eine tiefe Mädchenbeteiligung. Die geschlechterspezifische gymnasiale Typenwahl bleibt damit kantonal sehr unterschiedlich und scheint in keinem direkten Verhältnis mit der Mädchenbeteiligung im Gymnasium insgesamt zu stehen. Dafür hatte die Einführung des Typus D Ende der 1970er-Jahre in fast allen Kantonen einen Einfluss auf den Anstieg der Mädchenbeteiligung.

Mathematisch-naturwissenschaftlicher Typus $\mathrm{C}$

Aufgrund der bildungspolitischen Programmatik der "geschlechterblinden» (Rieger, 2001) Bildungsexpansion sind die kantonalen Differenzen beim Typus C einen genaueren Blick wert (Abb. 4). Der Typus C wurde zunächst schwach und verstärkt erst seit den 1960er-Jahren von Mädchen besucht. Die Mädchenbeteiligung stieg dann bis zur Abschaffung der Typengymnasien in den 1990er-Jahren permanent an. Die Unterschiede zwischen den Kantonen blieben verhältnismässig gross. Im Schuljahr 2000/01 findet sich zwischen ZG (16\%) und BS (45\%) die grösste Differenz, allerdings bei stark sinkenden Fallzahlen nach der formalen Abschaffung der gymnasialen Typen durch das Maturitätsanerkennungsreglement im Jahr 1995. Mit Blick auf die historische Entwicklung sind insbesondere die Kantone NE und TI interessant. Beide zeigen früh eine verhältnismässig hohe und ab Mitte der 1960er-Jahre eine stark steigende Mädchenbeteiligung im Typus C. Die anderen Kantone holten viel später und nur langsam auf. Gleichzeitig zeigt sich in diesen zwei Kantonen auch eine Konsolidierung der Mädchenbeteiligung im Typus C in den 1980er-Jahren bei rund 30\%. Eine Bildungsexpansionspolitik, die primär auf die Förderung männlicher Ingenieure und Naturwissenschaftler ausgerichtet war, hat in diesen Kantonen offenbar einen anderen Effekt erzielt, als anderswo. Ein Erklärungsversuch mittels vergleichbaren Strukturmerkmalen (z.B. Wirtschafts- und Bevölkerungsstruktur oder Konfession) läuft am Beispiel dieser zwei Kantone ins Leere.

\section{Abbildung 4}

Gymnasiale Mädchenbeteiligung im Typus C

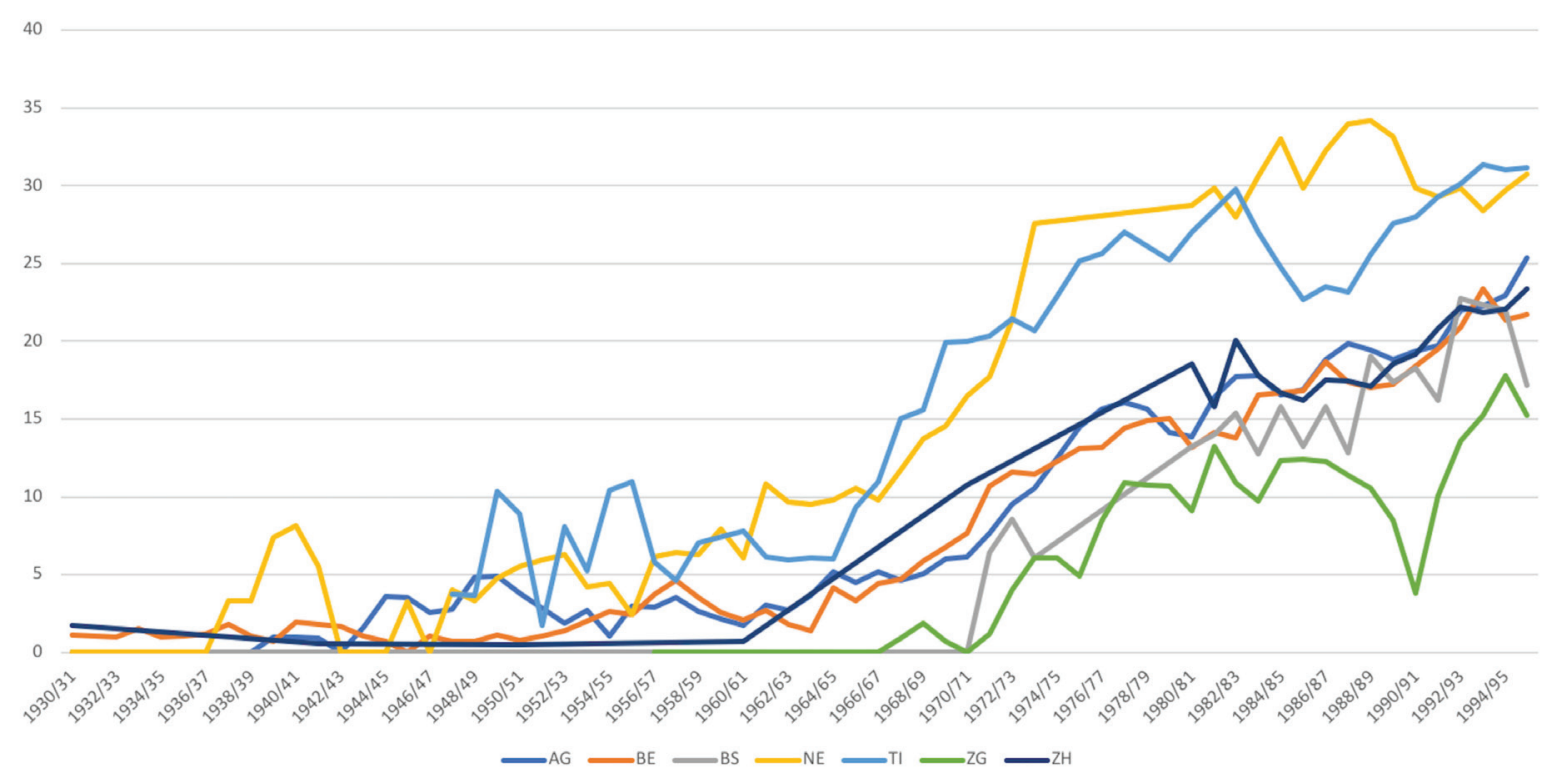

Bem. Daten in Prozent, 1930 bis zur MAR-Reform. 


\section{Bildungsexpansion und Mittelschulfinanzierung}

Der Ausbau der gymnasialen Mittelschulen setzt eine entsprechende Finanzierung voraus. Gezielte Investitionen in die Mädchenbildung wurden ab den 1970er-Jahren auch in Grundsatzpapieren der Erziehungsdirektorenkonferenz (EDK) eingefordert (EKF, 2001, S. 10). Eine Analyse der relativen Bildungsausgaben innerhalb der Sekundarschule II sowie der Pro-Kopf-Ausgaben nach Kanton für die Gymnasien für die hier untersuchten 13 Kantone zeigt, dass im Kontext der Bildungsexpansion in Relation zu anderen Schulstufen kein wesentlicher Anstieg der kantonalen Ausgaben in die Sekundarschule II erfolgte. Dies obwohl der Ausbau der Gymnasien einen zentralen Diskussionspunkt der Debatte darstellte. ${ }^{5}$ Gleichzeitig zeigt sich, dass bei einem forcierten Ausbau der Mädchenbeteiligung auch die entsprechende Finanzierung gewährleistet werden musste. In den untersuchten Innerschweizer Kantonen, in Abb. 5 am Beispiel SZ (rot), zeigt sich zunächst sowohl eine tiefe Mädchenbeteiligung sowie tiefe öffentliche Bildungsausgaben für die Mittelschulen. Dasselbe Muster trifft auch für andere Innerschweizer Kantone zu, die bis in die 1970er-Jahre klar segregierte Schulstrukturen auf der Sekundarstufe II aufwiesen. Gleichzeitig korrelieren hohe Bildungsausgaben für die Mittelschulen in einigen Fällen mit einer hohen Mädchenbeteiligung, gut sichtbar hier etwa in BS (grau), und bis in die 1960/70erJahre auch in SH und TI. Die hohen Differenzen der Mittelschulausgaben pro Kopf entsprechen für SZ und BS den hohen Unterschieden der Mädchenbeteiligung. Gerade im Kanton Schwyz sehen wir, wie ein relativer Investitionsschub in den 1970ern mit einem schnellen Wachstum der Mädchenbeteiligung zusammenhing. Eine wachsende Mädchenbeteiligung im Gymnasium setzt demnach die Bereitschaft voraus, entsprechende Angebote zur Verfügung zu stellen und zu finanzieren. Mehrausgaben allein sind andererseits kein hinreichendes Indiz um Veränderungen in der Beteiligung von Mädchen zu verstehen. So zeigt das Beispiel NE (blau), dass eine hohe Mädchenbeteiligung nicht mit vergleichsweise hohen Pro-Kopf-Ausgaben für die Mittelschule einhergehen muss.

\section{Abbildung 5}

Gymnasiale Mädchenbeteiligung und die Finanzierung der Mittelschulen

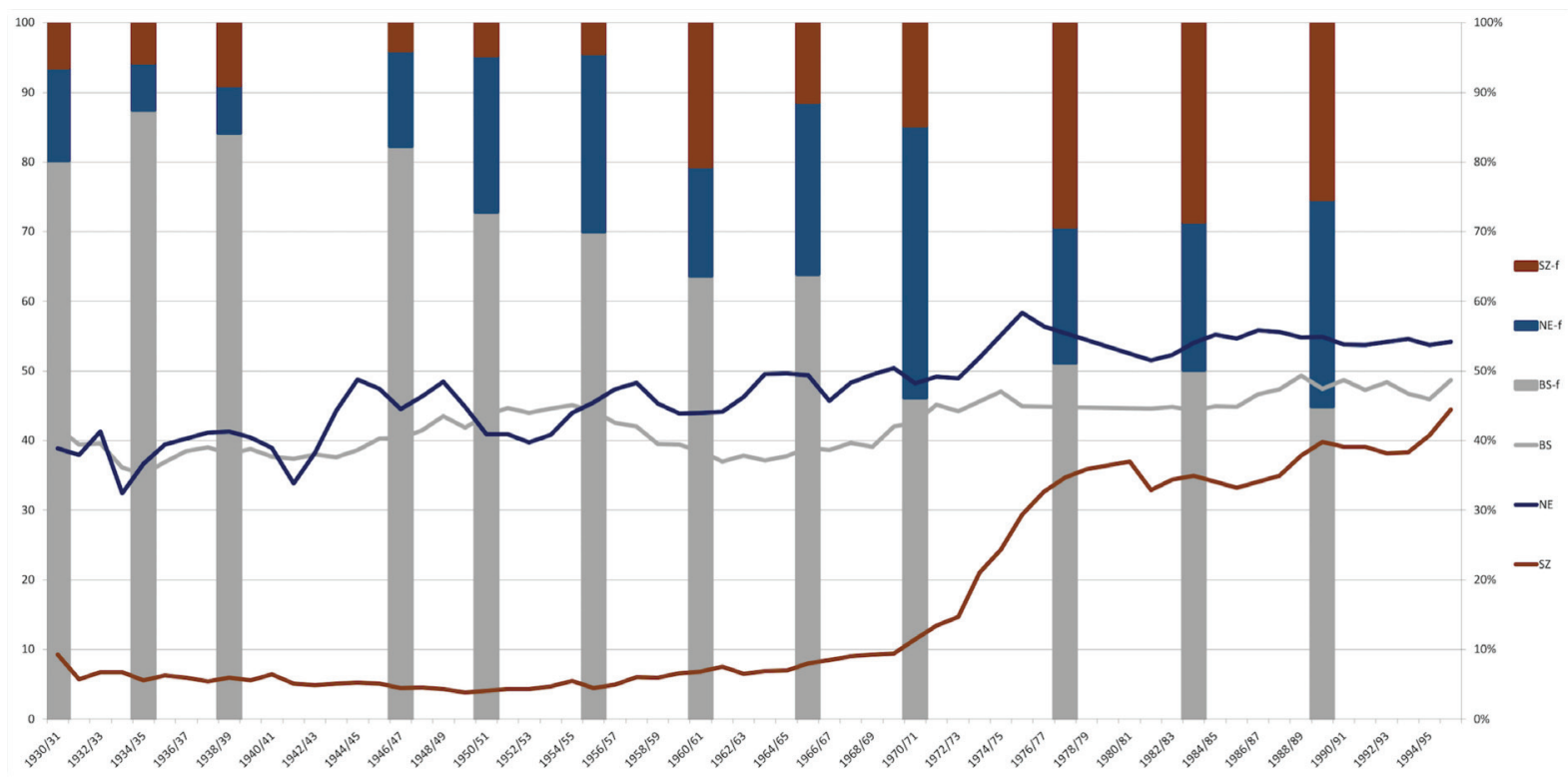

Bem. Kurven: Mädchenbeteiligung in Prozent.

Balken: absolute Mittelschulausgaben pro Einwohner*in in BS, NE und SZ im direkten Vergleich in Prozent.

5 Die absoluten Ausgaben pro Kopf stiegen gleichzeitig in allen Kantonen seit den 1960er-Jahren stark an. 


\section{Diskussion und Ausblick}

Die kantonalen Unterschiede in der Mädchenbeteiligung der gymnasialen Sekundarstufe II sind nach wie vor ein Phänomen, das politische Aufmerksamkeit erfährt. Dass nicht nur die kantonalen Unterschiede per se problematisiert werden, sondern auch die inzwischen in allen Kantonen höhere Präsenz von Mädchen, scheint gerade vor dem Hintergrund der langfristigen historischen Entwicklung paradox. Diese Entwicklungen wurden bislang nicht systematisch beschrieben und von der erziehungswissenschaftlichen Forschung nur marginal sozialhistorische Methoden der deskriptiven Statistik genutzt. Ausgehend von diesem wissenschaftlichen Desiderat und den bildungspolitischen Debatten, sind wir in diesem Artikel zwei zentralen Fragen nachgegangen: Wie verändert sich die Mädchenbeteiligung in der gymnasialen Sekundarstufe II im interkantonalen Vergleich in der Schweiz im Verlauf des 20. Jahrhunderts? Inwiefern gingen strukturelle Differenzierungen innerhalb der gymnasialen Sekundarstufe II mit einer veränderten Mädchenbeteiligung einher?

In einer längeren Phase nach der Zulassung von Mädchen zu gymnasialen Bildungsgängen seit dem frühen 20. Jahrhundert konnten wir eine vergleichsweise tiefe Mädchenbeteiligung bei einer langanhaltenden Disparität zwischen den Kantonen feststellen. Diese Disparität weist ihrerseits wieder typische kantonale Verläufe auf: Kantone mit einer stabil hohen Mädchenbeteiligung, solche mit einem kontinuierlichen Wachstum und schliesslich Kantone mit einer sehr tiefen Beteiligung und einer schnellen Öffnung seit den 1970er-Jahren. Diejenigen Kantone mit einer stabil hohen Mädchenbeteiligung sowie mit einer späten Öffnung zeichnen sich dabei durch einige Strukturmerkmale aus. Die beiden Gruppen unterscheiden sich betreffend Urbanisierungsgrad, Konfessionalität und Sprachregion (teilweise) deutlich voneinander. Im Gegensatz dazu wiesen die Kantone mit einem kontinuierlichen Wachstum der Mädchenbeteiligung eine beträchtliche strukturelle Heterogenität auf. Die Bedeutung der Typendifferenzierung liess sich in den Kantonen nicht mittels Strukturmerkmalen erklären. Diese Phase hoher kantonaler Disparität ist in einer zweiten Phase der Homogenisierung seit den 1980er-Jahren und einer Stabilisierungsphase seit den 2000er-Jahren gemündet.

Die Periode der Bildungsexpansion wies eine erhöhte Bereitschaft zur Mittelschulfinanzierung und damit einen Schub in der Angleichung der gymnasialen Mädchenbeteiligung zwischen den Kantonen auf. Gleichzeitig konzentrierte sich dieser Effekt insbesondere auf die kleineren Kantone mit bis dahin geringer Mädchenbeteiligung. Neben dem Ausbau bestehender Bildungsgänge wurden in diesem Zeitraum zuvor stark von Mädchen frequentierte Ausbildungsgänge als Maturitätstypen anerkannt. Der Typendifferenzierung innerhalb der Gymnasien ging gewissermassen eine Systemintegration voraus. Darüber hinaus kann die explizite Bildungsexpansionspolitik mit dem Ziel der Förderung der mathematisch-naturwissenschaftlichen Ausbildungsgänge als nur partiell erfolgreich betrachtet werden. Der Typus $\mathrm{C}$ blieb - wenn auch mit grossen kantonalen Unterschieden - männlich geprägt. Die «Geschlechterblindheit» der Bildungsexpansionspolitik war demnach kantonal sehr unterschiedlich ausgeprägt. Insgesamt blieb die Mädchenbeteiligung im Typus A/B für die Gesamtentwicklung prägend. Die Einführung neuer Typen hatte keinen unmittelbaren und dauerhaften Einfluss. Daraus folgt, dass die Bildungsexpansion der 1960/70er-Jahre allein keine hinreichende Erklärung bietet, da sie einen regional begrenzten Einfluss auf die steigende Mädchenbeteiligung in den Gymnasien aufweist. Diese Antwort auf die Frage nach der Bedeutung der Systemdifferenzierung bleibt folglich eine vorläufige. Studien zum Geschlechterverhältnis innerhalb der berufsbildenden Sekundarstufe II legen nahe, für die kantonalen Langzeitentwicklungen Bildungsalternativen systematisch zu erschliessen und darauf begründet, individuelle Laufbahnentscheidungen zu untersuchen. An dieser Stelle liegen denn auch die Grenzen der Aussagemöglichkeiten der bisher erschlossenen und vergleichbaren Datensätze. Geschlechterspezifische Langzeitreihen zu anderen als den gymnasialen Mittelschulen sind bislang nur eingeschränkt zugänglich. Der Fokus auf die Schulen und die Schüler*innenzahlen erlaubt darüber hinaus keine Rekonstruktion individueller Bildungsverläufe. Eine entsprechende Rekonstruktion über die Geburtenkohorten der (jeweils 10-jährigen) Volkszählungen liesse aber einen interkantonalen Vergleich von Bildungsinstitutionen nicht mehr zu. Wir haben bislang keine quantifizierenden Erklärungsversuche zur Bedeutung sozio-ökonomischer oder kultureller Variablen für die festgestellten kantonalen Unterschiede angeboten. Dies wollen wir nur bedingt mit Limitationen innerhalb der zugänglichen Langzeitdaten begründen (beispielsweise fehlender schulstatistischer Daten zum sozio-ökonomischen Status von Schüler*innen vor den 1960er-Jahren). Wir verstehen diese Studie als Angebot in zweierlei Richtungen: sowohl einer fundierten quantifizierenden Analyse zur Prüfung weiterer Hypothesen, als auch einer kontextualisierenden, die einzelnen Fälle betrachtenden, qualitativen Analyse. Nur durch eine umsichtige Kombination beider Perspektiven lässt sich nachvollziehbar machen, wie die institutionellen Rahmenbedingungen von Schule die Geschlechterdifferenzen und die Bildungschancen beeinflussen können. 


\section{Literatur}

Albisetti, J. C., Goodman, J., \& Rogers, R. (2010). Girls' secondary education in the Western World: From the 18th to the 20th century. Palgrave Macmillan.

Becker, R. \& Lauterbach, W (2016). Bildung als Privileg - Ursachen, Mechanismen, Prozesse und Wirkungen. In R. Becker \& W. Lauterbach (Hrsg.), Bildung als Privileg (S. 3-53). Springer.

Borkowsky, A. (2000). Frauen und Männer in der Berufsbildung der Schweiz. Schweizerische Zeitschrift für Bildungswissenschaften, 22(2), 279-294. https://doi.org/10.24452/sjer.22.2.4567

Breen, R., \& Jonsson, J.O. (2000). Analyzing educational careers: A multinomial transition model. American Sociological Review, 65(5), 754-773. https://doi.org/10.2307/2657545

Chuard, P., \& Grassi, V. (2020). Switzer-Land of Opportunity: Intergenerational Income Mobility in the Land of Vocational Education. SSRN Scholarly Paper. http://dx.doi.org/10.2139/ssrn.3662560

Criblez, L. (2001). Bildungsexpansion durch Systemdifferenzierung - Am Beispiel der Sekundarstufe II in den 1960er- und 1970er Jahren. Schweizerische Zeitschrift für Bildungswissenschaften, 23, 95-118. https://doi.org/10.24452/sjer.23.1.4594

Criblez, L. (2002). Gymnasium und Berufsschule: Zur Dynamisierung des Verhältnisses durch die Bildungsexpansion seit 1950. Traverse, 2, 29-40. http://dx.doi.org/10.5169/seals-22955

Crotti, C. (2005). Lehrerinnen - frühe Professionalisierung: Professionsgeschichte der Volksschullehrerinnen in der Schweiz im 19. Jahrhundert. Peter Lang.

Eberle, F. (2018). Die Maturitätsreform 1995. Intention, Evaluation der Wirkung und Anpassungsmassnahmen. In F. Imlig, L. Lehmann \& K. Manz (Hrsg.), Schule und Reform. Veränderungsabsichten, Wandel und Folgeprobleme (S. 213-227). Springer. https://doi.org/10.5167/uzh-150615

Eidg. Kommission für Frauenfragen [EKF] (2001). Mädchenbildung und Koeducation. In Eidg. Kommission für Frauenfragen (Hrsg.), Frauen Macht Geschichte. Zur Geschichte der Gleichstellung in der Schweiz 1848 - 2000.

Giudici, A. \& Manz, K. (2018). Knabenlernzeiten - Mädchenlernzeiten: Gleich, gleicher, ungleich? Genderspezifische Differenzierungen schulischen Wissens. Schweizerische Zeitschrift für Bildungswissenschaften, 40(3), 603-620. https://doi.org/10.24452/ sjer.40.3.5119

Helbig, M. (2013). Geschlechtsspezifischer Bildungserfolg im Wandel. Eine Studie zum Schulverlauf von Mädchen und Jungen an allgemeinbildenden Schulen für die Geburtsjahrgänge 1944-1986 in Deutschland. Journal for educational research online, 5(1), 141-183. https://www.waxmann.com/artikelART102718

Hodler, B. (2014). Junge Schule - lange Geschichte: Die neue Kantonsschule Aarau. hier + jetzt.

Hofstetter, R., Fontaine, A., Huitric, S., \& Picard, E. (2014). Mapping the discipline history of education. Paedagogica Historica, 50(6), 871-880. https://doi.org/10.1080/00309230.2014.948017

Imdorf, C., Sacchi, S., \& Wohlgemuth, K. (2014). How cantonal education systems in Switzerland promote gender-typical school-to-work transitions. Schweizerische Zeitschrift für Soziologie, 40(2), 175-196. http://dx.doi.org/10.7892/boris.67589

Jacobi, J. (2013). Mädchen- und Frauenbildung in Europa: Von 1500 bis zur Gegenwart. Campus.

Jey Aratnam, G. (2016). Der Talent-Turn und seine staatliche Begleitmusik: Migration und die Politik der Hochqualifizierung. In L. Criblez, C. Rothen \& T. Ruoss (Hrsg.), Staatlichkeit in der Schweiz: regieren und verwalten vor der neoliberalen Wende (S. 341-366). Chronos. https://doi.org/10.26530/OAPEN_625135

Joris, E. (2011). Liberal und eigensinnig: Die Pädagogin Josephine Stadlin - die Homöopathin Emilie Paravicini-Blumer: Handlungsspielräume von Bildungsbürgerinnen im 19. Jahrhundert. Chronos.

Kellerhals, K. (2010). Der gute Schüler war auch früher ein Mädchen: Schulgesetzgebung, Fächerkanon und Geschlecht in der Volksschule des Kantons Bern 1835-1897. Haupt.

Lelièvre, F. et Lelièvre, C. (1991). Histoire de la scolarisation des filles. Editions Nathan.

Lipset, S. M. (1967). Party systems and voter alignments: Cross-national perspectives. Free Press.

Martin, J., \& Goodman, J. (Eds.) (2011). Women and education: Major themes in education. Routledge.

McDaniel, A. (2010). Cross-National Gender Gaps in Educational Expectations: The Influence of National-Level Gender Ideology and Educational Systems. Comparative Education Review, 54(1), 27-50. https://doi.org/10.1086/648060

Mayeur, F. (1977). L'enseignement secondaire des jeunes filles sous la troisième république. Presses de la fondation nationale des sciences politiques.

Monnier, A. (2018). Le temps des dissertations: Chronique de l'accès des jeunes filles aux études supérieures (Genève, XIXe-XXe). Droz.

Mantovani Vögeli, L. (1994). Fremdbestimmt zur Eigenständigkeit: Mädchenbildung gestern und heute. Rüegger.

Ragni, T. (2014). Chancengerechtigkeit durch Bildungsinvestitionen? bildungspolitik. Zeitschrift für Bildung, Erziehung und Wissenschaft, 185. http://vpod-bildungspolitik.ch/?p=1473

Renold, U. (1998). "Wo das Männliche anfängt, da hört das Weibliche auf»!: Frauenberufsbildungsdiskussionen im Spiegel der sozioökonomischen Entwicklung (1860-1930). Selbstverlag.

Rieger, A. (2001). Bildungsexpansion und ungleiche Bildungspartizipation am Beispiel der Mittelschulen im Kanton Zürich, 1830 bis 1980. Schweizerische Zeitschrift für Bildungswissenschaften, 23(1), 41-72. https://doi.org/10.24452/sjer.23.1.4596

Rogers, R. (2010). L'éducation des filles. Dans F. Jacquet-Francillon, R. Renaud-d'Enfert et R. Loeffel (dir.), Une histoire de l'école. Anthologie de l'éducation en France. XVIIIe-XXe siècle (p. 165-171). Retz.

Ruoss, T. (2013). Historical Change in the Production and Legitimisation of Education Statistics in Switzerland. European Educational Research Journal, 12(1), 95-107. https://doi.org/10.2304/eerj.2013.12.1.95

Schweizerische Koordinationsstelle für Bildungsforschung [SKBF] (2018). Bildungsbericht Schweiz 2018. Schweizerische Koordinationsstelle für Bildungsforschung.

Ullmann, L. (2019). Der Zugang zur gymnasialen Bildung für Mädchen: Eine akteurzentrierte Betrachtung gymnasialer Institutionen. Unveröffentlichte Masterarbeit, Institut für Erziehungswissenschaft der Universität Zürich. 
Vonlanthen, A., Lattmann, U. P., \& Egger, E. (1978). Maturität und Gymnasium. Ein Abriss über die Entwicklung der eidgenössischen Maturitätsordnungen und deren Auswirkungen auf das Gymnasium. Haupt.

Zangger, C. \& Becker, R. (2016). Die Bildungsexpansion in der Schweiz - eine Reanalyse unter besonderer Berücksichtigung geschlechtsspezifischer Bildungschancen. Swiss Journal of Sociology, 42(3), 428-441. https://doi.org/10.1515/sjs-2016-0019

Zymek, B., Neghabian, G., \& Ziob, L. (2005). Sozialgeschichte und Statistik des Mädchenschulwesens in den deutschen Staaten 1800-1945: Bd.2, Teil 3. Vandenhoeck \& Ruprecht.

Quellen

Bundesamt für Statistik (BfS):

Sekundarstufe II: Lernende nach Maturitätstyp (A, B, C, D, E), Kanton und Geschlecht, 1980/01 - 2003/04.

Sekundarstufe II: Lernende nach Bildungstyp, Kanton und Geschlecht, 1980/01 - 2016/17.

Bildungsgeschichte Schweiz: https://www.bildungsgeschichte.ch/de/

Bestand/Projekt: Bildung in Zahlen

Bildungssystematik: Mittelschule

Historische Statistik der Schweiz (HSSO): https://hsso.ch/

B.6b.: Haushaltungen nach Kantonen 1850-1990: mittlere Personenzahl pro Haushaltung

E.1b.: Geschätzte Fünfjahres-Wanderungsbilanz nach Kantonen 1910-1985.

U.33. Ausgaben der Kantone: Funktionale Gliederung nach Kantonen 1930-1989.

Schlagworte: Mädchenbildung; Historische Statistik; Bildungspolitik; Gymnasium; Soziale Ungleichheit

\section{Étudiantes au lycée : une comparaison intercantonale de l'inégale fréquentation de cette institution par les filles en Suisse au $20^{e}$ siècle}

Résumé

Cet article présente, sur la base de données statistiques historiques, l'évolution de la fréquentation féminine des établissements gymnasiaux du secondaire II en Suisse au cours du $20^{\mathrm{e}}$ siècle. L'article présente les grandes différences historiques entre les cantons et déduit trois phases différentes de fréquentation du lycée (gymnase) par les élèves féminines. Dans un deuxième temps, des explications des différences identifiées seront développées en fonction de la différenciation structurelle des lycées et l'importance des contextes institutionnels pour les différences entre les genres à l'école sera discutée.

Mots-clés : Éducation des filles ; statistiques historiques ; politique éducative ; lycée ; inégalité sociale

\section{Studentesse al liceo: un confronto intercantonale sulle disugualianze della frequentazione delle ragazze nella Svizzera del XX secolo}

Riassunto

Questo articolo esplora una vasta base di dati statistici storici per tracciare l'evoluzione della frequentazione femminile ai licei svizzeri nel corso del XX secolo. Innanzitutto, vengono ricostruite le profonde differenze storiche fra i cantoni, dalle quali si deducono tre fasi che caratterizzano la presenza delle ragazze nei licei. Su queste basi lo studio sviluppa delle prime spiegazioni per queste disparità, considerando in particolare le varietà strutturali. Questo ci permette di discutere l'importanza dei contesti istituzionali per le disugualianze di genere nella scuola.

Parole chiave : Istruzione femminile; statistica storica; politica scolastica; liceo; disuguaglianza sociale 


\section{Girls in higher secondary education: a comparison of the unequal participation of girls in Switzerland in the 20th century}

Summary

This article presents the development of girls' participation in higher secondary schools in Switzerland during the 20th century based on comprehensive long-term statistical data. First of all, the large historical differences between Swiss Cantons are presented and three phases of the varying participation of girls in grammar school are derived. Explanations for the differences identified are developed with a view to the structural differentiation of higher secondary education. This allows us to discuss the importance of institutional frameworks for gender differences in schools.

Keywords: Girls' education; historical statistics; education policy; grammar school; social inequality

Thomas Ruoss arbeitet als Post-Doc am Lehrstuhl für Berufsbildung der Universität Zürich. Forschungsschwerpunkte: Bildungspolitikforschung, historische Bildungsforschung, Statistikgeschichte, Ökonomie und Bildung; Institut für Erziehungswissenschaft, Universität Zürich, Kantonsschulstrasse 3, CH-8001 Zürich. E-Mail: truoss@ife.uzh.ch

Lars Heinzer ist wissenschaftlicher Mitarbeiter im Forschungsprojekt «Bildung in Zahlen». Forschungsschwerpunkte: Historische Bildungsforschung, Historische Bildungsstatistik mit speziellem Fokus auf Pflege- und Landwirtschaftsschulen; Institut für Erziehungswissenschaft, Universität Zürich, Freiestrasse 136, 8001 Zürich. E-Mail: lars.heinzer@ife.uzh.ch 